REVISTA DE LA ESCUela de CIENCIAS DE LA EDUCACIÓN, AÑo 15, NRO. 14, VOL. 1, ENERO A JUNIO DE 2019. PÁGINAS 26-35. ISSN 1851-6297 (DESDE DICIEMBRE DE 2006 A DICIEMBRE DE 2017). ISSN 2362-3349 (EN LÍNEA). INCIDENCIA DE LOS FACTORES SOCIO-AFECTIVOS EN EL APRENDIZAJE DEL INGLÉS. LEYDY FERNANDA BONILLA JOJOA · MARIO DÍAZ VILLA.

\title{
INCIDENCIA DE LOS FACTORES SOCIO-AFECTIVOS EN EL APRENDIZAJE DEL INGLÉS
}

\author{
Leydy Fernanda Bonilla Jojoa* \\ Universidad Santiago de Cali, Colombia \\ leydy.bonilla00@usc.edu.co \\ Mario Díaz Villa**. \\ Universidad Santiago de Cali, Colombia \\ mdiazvilla@gmail.com
}

Recibido: 26/20/2018 Aceptado: 10/12/2018

\begin{abstract}
Resumen
El propósito de este artículo es realizar un análisis de los factores motivación, ansiedad y autoestima en el aprendizaje del inglés como lengua extranjera. Se revisaron de manera crítica las nociones, tipologías y perspectivas relacionadas con cada uno de estos factores socio-afectivos y su incidencia en el aprendizaje de una lengua extranjera. Se encontró que éstos influyen notoriamente en el éxito o fracaso en el proceso de aprendizaje de un idioma y que entran en relaciones directas o inversas debido a su relativa autonomía. En adición a los factores personales se describieron los factores contextuales y se asumió como determinante el papel que juega el profesor como agente pedagógico, ya que éste incide de alguna manera en el comportamiento y actitud de los estudiantes en el aula de clase. Finalmente, se estableció que los factores personales y contextuales no operan de manera aislada, sino que se presuponen mutuamente. El artículo tiene un gran significado para los docentes, ya que les permite reconocer la importancia que juegan los factores socio-afectivos en el aprendizaje de un idioma y, por lo tanto, incorporar en su labor estrategias flexibles que permitan desarrollar un ambiente favorable para el aprendizaje.
\end{abstract}

Palabras clave: Motivación - Autoestima - Ansiedad - Aprendizaje - Lengua extranjera.

\section{Abstract}

The purpose of this paper is to perform an analysis of motivation, anxiety and self-esteem factors in the learning of English as a foreign language. The notions, typologies and perspectives of each one of these socio-affective factors and their impact on the learning of a foreign language were critically reviewed. It was found that those factors had a marked influence on the success or failure in the process of learning a language and that, entering into a direct or inverse relationship due to their relative autonomy. In addition to the personal factors, the contextual factors were also described, and it was assumed that the role played by the teacher as a pedagogical agent, since it is related to the

* Licenciada en Lenguas Extranjeras Inglés y Francés de la Universidad Santiago de Cali y Maestrante en Educación de la misma Universidad. Actualmente es docente de inglés hora catedra en la Institución Universitaria Antonio José Camacho.

** Licenciado en Literatura e Idiomas. Estudios de maestría en Lingüística de la Universidad de Valle y PhD en Educación de la Universidad de Londres. Actualmente se desempeña como docente de la Universidad Santiago de Cali tiempo completo. 
REVISTA DE LA ESCUELA DE CIENCIAS DE LA EDUCACIÓN, AÑo 15, NRO. 14, VOL. 1, ENERO A JUNIO DE 2019. PÁGINAS 26-35. ISSN 1851-6297 (DESDE DICIEMBRE DE 2006 A DICIEMBRE DE 2017). ISSN 2362-3349 (EN LÍNEA). INCIDENCIA DE LOS FACTORES SOCIO-AFECTIVOS EN EL APRENDIZAJE DEL INGLÉS. LEYDY FERNANDA BONILLA JOJOA · MARIO DÍAZ VILLA.

form and behavior of the students in the classroom. Consequently, it was established that the personal and contextual factors do not operate in isolation, but they presuppose each other. The paper has a great significance for teachers, as it allows them to recognize the importance of socioaffective factors in learning a language and make them think that incorporate flexible strategies in their work allow the development of a favorable environment for learning.

Keywords: Motivation - Self - Esteem - Anxiety - Learning and Foreign language.

\section{Introducción}

Esta investigación tuvo por objeto analizar la incidencia de los factores personales como la motivación, la ansiedad, la autoestima, y el papel que juega el profesor como actor pedagógico en el proceso de aprendizaje de una Lengua Extranjera (L.E). El proyecto surgió del problema que se observó en estudiantes del Centro de Idiomas de la Institución Universitaria Antonio José Camacho ubicado en la sede sur de la ciudad de Cali (Colombia), los cuales presentaron bajo rendimiento académico en los cursos de inglés 1 A1.1. En un grupo de 20 estudiantes, el $50 \%$ presentaron estas dificultades. Es común encontrar comentarios de los estudiantes sobre su actitud hacia esta asignatura, y expresiones de desmotivación o indisposición, tales como: "Esto no es para mi", "No quiero participar", "No me siento seguro en un idioma que no es mío", "Me da pena". Estas afirmaciones, por lo general, surgen en grupos de estudiantes con desempeño básico o bajo en el aprendizaje del inglés como lengua extranjera. El desinterés por el aprendizaje, la alta deserción de los cursos, la visión de la asignatura como un requisito para graduarse y el no contemplar la necesidad de aprender un segundo idioma, son los motivos que han influenciado el presente estudio. Esta investigación toma como categorías de análisis los factores socioafectivos y el papel del docente, sus interdependencias y las determinaciones que pueden presentarse entre unos y otros. Sin embargo, el estudio solo toma como objeto tres factores personales y restringe los contextuales al papel que juega el maestro.

El artículo analiza los factores socio-afectivos en el aprendizaje de inglés como L.E. El punto de partida son los planteamientos de autores como Gardner (1985, 2010), Horwitz \& Cope (1986), Dörnyei ,2010,2008, 1998, 1994) Deci \& Ryan (2000) y Arnold \& Brown (2006) quienes han determinado que el aprendizaje y el desempeño del estudiante de una lengua extranjera se ven afectados, de una u otra manera, por factores internos y externos. Para que el aprendizaje de una nueva lengua se realice con total éxito se requiere no solo de una buena metodología implementada por parte del docente. También, resulta indispensable que el maestro conozca las actitudes, las expectativas, las motivaciones y demás sentimientos que los estudiantes tengan frente al aprendizaje de una L.E. En este sentido, es significativo aclarar que los factores socio-afectivos tiene un fuerte vínculo en la interacción pedagógica maestroestudiante, ya que no operan de manera aislada, sino que interactúan y se interrelacionan.

En el aprendizaje de una L.E los factores socio-afectivos se consideran componentes claves para que un estudiante asimile de manera significativa conocimientos que el maestro le brinda en el aula de clase; pues éstos juegan un papel importante en todas las actividades de la vida cotidiana. La motivación es considerada desde la Psicología como un factor que favorece o debilita el comportamiento para llegar a la meta. Hilgard, Atkinson \& Atkinson (1979) la definen como "un conjunto de factores que vigorizan la conducta y le dan una dirección” (Hilgard, Atkinson \& Atkinson, 1979, p. 281). Es importante señalar que la motivación, el éxito y el rendimiento académico son conceptos que están estrechamente relacionados (Peiteado \& López, 2017). Esto significa que la motivación es intrínseca al proceso de aprendizaje de una lengua extranjera y ha sido aceptada como un factor clave que influye en el proceso aprendizaje de una segunda lengua (L2) y de una L.E. En este sentido, Dörnyei (1998) afirma que:

sin la motivación suficiente, incluso las personas con las habilidades más notables no pueden lograr sus objetivos a largo plazo, y tampoco los planes de estudios adecuados y la buena enseñanza son suficientes para asegurar el rendimiento por parte de los estudiantes (Dörnyei, 1998, p. 117).

Sin embargo, es necesario precisar que la motivación no es el único factor socio-afectivo que influye en el proceso de aprendizaje de una L.E. Al respecto, Arnold, J. y Brown D. (2006) señalan que:

La comprensión de la función que la afectividad cumple en el aprendizaje de idiomas resulta significativa por dos motivos. En primer lugar, la atención a los aspectos afectivos puede lograr una mayor eficacia en el aprendizaje de idiomas. [...] Por otro lado, al estimular diferentes factores emocionales positivos como la autoestima, la empatía o la motivación, se facilita extraordinariamente el proceso de aprendizaje de idiomas (Arnold y Brown 2006, p. 257). 
REVISTA DE LA ESCUELA DE CIENCIAS DE LA EDUCACIÓN, AÑo 15, NRO. 14, VOL. 1, ENERO A JUNIO DE 2019. PÁGINAS 26-35. ISSN 1851-6297 (DESDE DICIEMBRE DE 2006 A DICIEMBRE DE 2017). ISSN 2362-3349 (EN LÍNEA). INCIDENCIA DE LOS FACTORES SOCIO-AFECTIVOS EN EL APRENDIZAJE DEL INGLÉS. LEYDY FERNANDA BONILLA JOJOA · MARIO DÍAZ VILLA.

Además de los factores socioafectivos mencionados existen otros como la personalidad, las creencias y emociones que intervienen en el proceso de aprendizaje. Autores como Villegas, García-Santillán \& Escalera-Chávez (2016) se refieren a los factores personales y cognitivos que inciden sobre el aprendizaje entre los cuales pueden mencionarse, de manera general, los siguientes: la edad, género, experiencias de aprendizaje, la inteligencia, las habilidades, estrategias y actitudes. Los factores socio-afectivos juegan un papel trascendental, debido a que generan estímulos y dominio en todo aquello que se realiza, y permiten que el aprendiz pueda alcanzar los objetivos propuestos y avanzar intelectualmente, sin señalar un área específica de conocimiento.

El artículo se divide en tres secciones: en la primera sección se abordará la motivación en términos de su definición, clasificación y las diferentes perspectivas de análisis del factor con respecto al aprendizaje de la L.E; en la segunda se tratará la autoestima en el aprendizaje de un segundo idioma y su relación con la ansiedad. En la tercera se conceptualizará el término de ansiedad y sus tipologías. Estos constructos permitirán identificar la incidencia de los factores socio-afectivos en el proceso de aprendizaje de un idioma.

\section{Una mirada de la motivación en el aprendizaje de una L.E}

Abordar el concepto de motivación no es tarea fácil pues en él convergen una multiplicidad de factores tanto internos como externos que condicionan el comportamiento del individuo, asociados a lo personal, lo contextual, lo social y sus interrelaciones. Es por esto que no se puede brindar una definición formal de este constructo pues ha sido relacionado con diversas variables que median en la conducta humana. Como lo afirman Navarro \& Jiménez (2018) "La razón por la cual la motivación se conceptualiza en términos abstractos es probablemente debido al hecho de que hay diferentes tipos de motivación" (Navarro \& Jiménez, 2018, p. 72). No obstante, este factor ha sido tratado a través de los tiempos por medio de una variedad de teorías y enfoques que están asociados con las experiencias de éxito o fracaso que determinan la conducta futura, la autoconfianza, la auto-eficiencia, y factores ligados a la personalidad (Weiner, 1979; Clément, 1980; Covington, 1992; Bandura, 2001; Maslow 1970, 2016).

En el campo de la psicología, la motivación también ha sido asumida como un proceso interno que puede estar influenciado tanto por aspectos externos como internos. La motivación como objetivo posee una dirección determinada (Abascal, Cantero \& Martínez-Sánchez, 2002; Murado, 2010). Así mismo se ha definido como: "La compleja integración de procesos psíquicos que efectúa la regulación inductora del comportamiento. Pues determina la dirección (hacia el objeto-meta buscado o el objeto-evitado) la intensidad y el sentido (de aproximación o evitación) del comportamiento" (Serra, 2008, p. 52). Lo anterior implica valorar la motivación como un componente determinante en el comportamiento de las personas para el logro de una meta precisa. Sin embargo, existe un abanico de posibilidades que pueden intervenir para que la persona tome una decisión que la impulse a llegar al objetivo de manera exitosa o, por el contrario, la lleve al fracaso. Al respecto Dörney \& Ushioda (2013) plantean que:

La experiencia de motivación de la mayoría de la gente en la vida real es bastante más compleja que la simple percepción de estados binarios de causa y efecto antes y después de una tarea o un evento. La motivación para hacer algo usualmente evoluciona gradualmente, a través de un complejo proceso mental que involucra planificación inicial establecimiento de metas, formación de intenciones, generación de tareas, implementación de acciones, control de acciones, evaluación de resultados. Estas diferentes subfases de la motivación pueden asociarse con diferentes motivos (Dörney \& Ushioda, 2013, p. 6).

Las contribuciones de la psicología a la compresión de la motivación en el proceso educativo han sido fundamentales para un mejor entendimiento de ésta, sobre todo, cuando se trata de una lengua extranjera. Peiteado \& López (2017) afirman que "la motivación impulsa a las personas a iniciar el aprendizaje de una lengua extranjera, influye en el mantenimiento de dicha conducta y juega un rol importante si el proceso de aprendizaje se torna difícil" (Peiteado \& López, 2017, p. 133). Como se ha señalado anteriormente, la conducta de las personas es condicionada por múltiples factores tales como los anhelos, los deseos, las habilidades, las emociones y los sentimientos que cada una posee internamente. En el caso del aprendizaje del inglés, o de cualquier otra asignatura, los aprendices presentan rasgos diferenciales. Por lo tanto, se hace necesario conocer sus necesidades e intereses para así generar un ambiente que motive el aprendizaje. Debido a la necesidad de comprender este constructo en mayor profundidad se han configurado unos modelos que tratan de abarcar las variables independientes que intervienen en el aprendizaje de una lengua extranjera.

Los trabajos realizados por Gardner \& Lambert (1972) han permitido construir un modelo psicosocial 
REVISTA DE LA ESCUELA DE CIENCIAS DE LA EDUCACIÓN, AÑo 15, NRO. 14, VOL. 1, ENERO A JUNIO DE 2019. PÁGINAS 26-35. ISSN 1851-6297 (DESDE DICIEMBRE DE 2006 A DICIEMBRE DE 2017). ISSN 2362-3349 (EN LÍNEA). INCIDENCIA DE LOS FACTORES SOCIO-AFECTIVOS EN EL APRENDIZAJE DEL INGLÉS. LEYDY FERNANDA BONILLA JOJOA · MARIO DÍAZ VILLA.

enfocado al aprendizaje de las lenguas. Gardner (2010) ha precisado que la motivación es una herramienta potencial y determinante al momento de aprender una lengua extranjera. Así mismo ha determinado que un "aprendiz motivado" cuenta con tres características específicas, que conllevan que tenga éxito en su aprendizaje. Dichas características son:

Deseo por aprender la lengua o el idioma.

Disposición para esforzarse en medio del proceso o actividad de aprendizaje.

Disposición para sostener la actividad de aprendizaje.

Entre los aportes que Gardner (2010) resalta en el modelo psicosocial está la distinción entre motivación integradora y motivación instrumental. La motivación integradora hace referencia al interés que tiene el aprendiz por interactuar con un grupo de personas que hable un determinado idioma. La motivación instrumental obedece a un interés o deseo práctico, es decir, hace referencia a la búsqueda de un mejor empleo, un diploma o algún reconocimiento social (Gardner, 2010).

Desde la perspectiva de las teorías cognitivas de la motivación, Dörnyei (1994 [2010]) señala que la motivación implica procesos cognitivos individuales, recoge los planteamientos tenidos en cuenta en el modelo psicosocial, y añade otros componentes trabajados en teorías desarrolladas en las corrientes cognitivas y en la psicología educativa. Es importante resaltar que desde la perspectiva psicosocial la teoría planteada por Gardner (1985) sobre el aprendizaje de las lenguas extranjeras aportó a la compresión del constructo motivación psicológica y sociológicamente. Ahora bien, con el surgimiento de nuevos estudios realizados en la década de los años 90 se vio la necesidad de pensar la motivación desde otras perspectivas.

En el modelo planteado por Dörnyei (1994 [2008]) la motivación se divide en tres niveles (1), los cuales contienen variables que un estudiante puede tener al momento de aprender un idioma. Además de incorporar la motivación instrumental e integradora dentro del nivel de lengua, Dörney (1994 [2008]) resalta la importancia de las actitudes y características propias de cada estudiante en relación con las variables afectivas autoestima y ansiedad. Asimismo, alude al papel del docente como un agente motivador del aprendizaje. Esto significa que éste es considerado un actor clave, ya que tiene el poder de modificar conductas al activar y dinamizar la interacción con sus estudiantes, y lograr que estos desarrollen el interés por el aprendizaje de la lengua. Esta teoría considera otros factores externos importantes a analizar dentro del proceso de aprendizaje de las lenguas, tales como el curso y el grupo.

En adición a los modelos anteriores se puede mencionar la teoría de la autodeterminación, la cual no solo aborda la conceptualización de la motivación a partir de los aspectos intrínsecos y extrínsecos, sino que amplía esta clasificación con una categoría denominada amotivación que puede considerarse como motivación cero. Para Deci y Ryan (2000) la motivación intrínseca es un constructo de orden natural que está relacionado con el interés, la curiosidad, el dominio, y la asimilación, que responden a un goce y que son vitales en el desarrollo cognitivo y social. Por su parte, Vallerand (1997) establece tres tipos de motivación intrínseca: la motivación para aprender, la cual está asociada a la satisfacción y al placer de aprender algo nuevo; la motivación hacia el logro, que comprende el agrado de participar en una actividad por un reto personal que le permita al aprendiz lograr el alcance de la meta o crear un nuevo conocimiento. Este tipo de motivación se centra más en el proceso y no en los resultados. Finalmente está la motivación de experiencias de estimulación, referida al placer que produce realizar actividades que generan sensaciones agradables.

El concepto de motivación extrínseca se refiere al "rendimiento de una actividad para lograr algunos resultados" (Deci y Ryan, 2000, p.71). Estos autores proponen cuatro tipos de motivación extrínseca (2), los cuales obedecen a fines prácticos para el alcance de la conducta esperada. En otras palabras, se trata de cumplir para obtener algo a cambio. Esta teoría fue abordada por Noels, Clément y Vallerand (2002) en el contexto de enseñanza-aprendizaje de segundas lenguas y fue considerada muy útil, ya que comprende ampliamente los factores tanto intrínsecos como extrínsecos en el aprendizaje, y cómo estos pueden variar según la intencionalidad que el estudiante tenga para aprender una lengua extranjera. En esta misma dirección Peiteado \& López (2017) afirman que:

El aprendizaje de una segunda lengua requiere asimilación, persistencia, esfuerzo intelectual sostenido y dedicación, conductas que no se manifiestan si se está desmotivado. Variables individuales, contextuales y pedagógicas son constructos que pueden enriquecer o dificultar la interacción entre motivación y aprendizaje (Peiteado \& López, 2017, p. 131). 
REVISTA DE LA ESCUeLA dE CIENCIAS DE LA EDUCACIÓN, AÑo 15, NRO. 14, VOL. 1, ENERO A JUNIO DE 2019. PÁGINAS 26-35. ISSN 1851-6297 (DESDE DICIEMBRE DE 2006 A DICIEMBRE DE 2017). ISSN 2362-3349 (EN LÍNEA). INCIDENCIA DE LOS FACTORES SOCIO-AFECTIVOS EN EL APRENDIZAJE DEL INGLÉS. LEYDY FERNANDA BONILLA JOJOA · MARIO DÍAZ VILLA.

En síntesis, la motivación no es un constructo que opera de manera intrínseca, debido a que son muchas las variables que inciden en ella, ya sean factores personales o contextuales que no permiten tener una compresión amplia del alcance que esta pueda llegar a tener. Sin embargo, la proliferación de las teorías ha contribuido al despliegue de tipologías que surgen y dan explicaciones acerca de su origen de la motivación, hasta llegar a sus fines prácticos.

\section{La autoestima en el aprendizaje de una L.E y su relación con la ansiedad}

La autoestima es considerada como una variable socio-afectiva que influye notoriamente en el aprendizaje. Aparte de las características de personalidad que pueden llegar a tener los estudiantes, se ha comprobado que este componente incide en el aprendizaje de la lengua extranjera, debido a que depende del docente, y de las estrategias y metodologías empleadas en fortalecer o debilitar la autoestima en los estudiantes respecto del proceso de aprendizaje del idioma. Arnold y Brown (2006) afirman que la autoestima:

Está relacionada con las inevitables evaluaciones que una persona realiza sobre su propia valía. Es un requisito fundamental para que las actividades cognitivas y afectivas tengan éxito. Obtenemos nuestras nociones de autoestima de nuestras experiencias internas y de nuestra relación con el mundo externo (Arnold y Brown, 2016, p. 266).

Este factor no solo se determina por la evaluación que se realiza de nosotros mismos, sino que tiene una significación social. Comprender los alcances que puede llegar a aportar el asociar la autoestima con el rendimiento garantiza la obtención de bases que fortalezcan no solo los aspectos emocionales sino, también, los cognitivos. Al respecto, investigaciones como las de Marins de Andrade \& Guijarro Ojeda (2010); Koosha, Ketabi, \& Kassaian (2011), y Rodríguez-Pérez (2012), han establecido la importancia que tiene reconocer la autoestima para mejorar los procesos de enseñanza-aprendizaje y, de esta forma, movilizar a los estudiantes al alcance de la lengua meta.

En el proceso de aprendizaje de una lengua nueva intervienen una serie de factores. Entre ellos están la motivación, la autoestima y la ansiedad. Por lo tanto, se hace necesario que los docentes consideren dentro de sus prácticas actividades que generen estímulos y permitan que los estudiantes ganen confianza de sí mismos y de sus habilidades lingüísticas, de lo contrario, estos pueden sentir que el aprendizaje de un idioma no es algo que este dentro de sus aptitudes o dominio. Así, las malas experiencias, o el inicio del proceso de aprendizaje de una lengua nueva, pueden provocar en los estudiantes limitaciones en el momento de interactuar o participar, debido a que conducen a cometer errores de nivel gramatical o de pronunciación. Al respecto, Carrón (2013) plantea que:

Los alumnos, sobre todo los adultos, traen a la clase una gran variedad de prejuicios sobre el aprendizaje de segundas lenguas. Estos prejuicios parten de la opinión que los alumnos tienen sobre sus propias habilidades en general o sobre la opinión particular en el caso del aprendizaje de idiomas por los resultados obtenidos en experiencias anteriores (Carrón, 2013, p. 278).

Los profesores de idiomas tienen el desafío de lograr desmitificar creencias que traen consigo algunos estudiantes al momento de aprender un idioma. En el momento que los estudiantes sienten que no tienen las competencias ni las habilidades para lograrlo, la autoestima puede verse amenazada por la ansiedad, ya que al presentarse inseguridad puede generarse una relación entre la baja autoestima y la ansiedad. En este sentido, Arnold (2000) considera que "en un sentido, la ansiedad y la autoestima son dos caras de una moneda afectiva, una que impide el aprendizaje y otra que la favorece" (Arnold, 200, p. 17).

Desde la misma perspectiva, Zare \& Riasati (2012) y Rubio-Alcalá (2017) manifestaron que existen relaciones interdependencia que se generan entre la autoestima y la ansiedad. No obstante, la autoestima es un concepto multifactorial que no se puede limitar ni correlacionar únicamente con la ansiedad. Mercer \& Williams (2014) asocian la autoestima y el auto-concepto ${ }^{3}$ con factores personales (la ansiedad), familiares, sociales y académicos entre otros. Esto significa, que la autoestima no solo comprende lo personal o se relaciona con éste. Vázquez \& Pérez (2016) plantean que "existe un nivel de ansiedad que es normal, denominado umbral emocional" (Vázquez \& Pérez, 2016, p.113). En otras palabras, la tipología de la ansiedad puede determinar si se ve o no 
REVISTA de LA ESCUela de CIENCIAS de LA EDUCACIÓN, AÑo 15, NRO. 14, VOL. 1, ENERO A JUNIO dE 2019. PÁGINAS 26-35. ISSN 1851-6297 (DESDE DICIEMBRE DE 2006 A DICIEMBRE DE 2017). ISSN 2362-3349 (EN LÍNEA). INCIDENCIA DE LOS FACTORES SOCIO-AFECTIVOS EN EL APRENDIZAJE DEL INGLÉS. LEYDY FERNANDA BONILLA JOJOA · MARIO DÍAZ VILLA.

afectado el individuo en alguna actividad. En este sentido, si se habla de una ansiedad de estado transitorio, ésta no necesariamente llegará a causar resultados perjudiciales. Para Vázquez \& Pérez (2016) los estudiantes pueden presentar una alta autoestima y niveles de ansiedad dentro de lo normal que no perturban su proceso de aprendizaje. La siguiente figura muestra las relaciones estructurales entre autoestima y ansiedad.

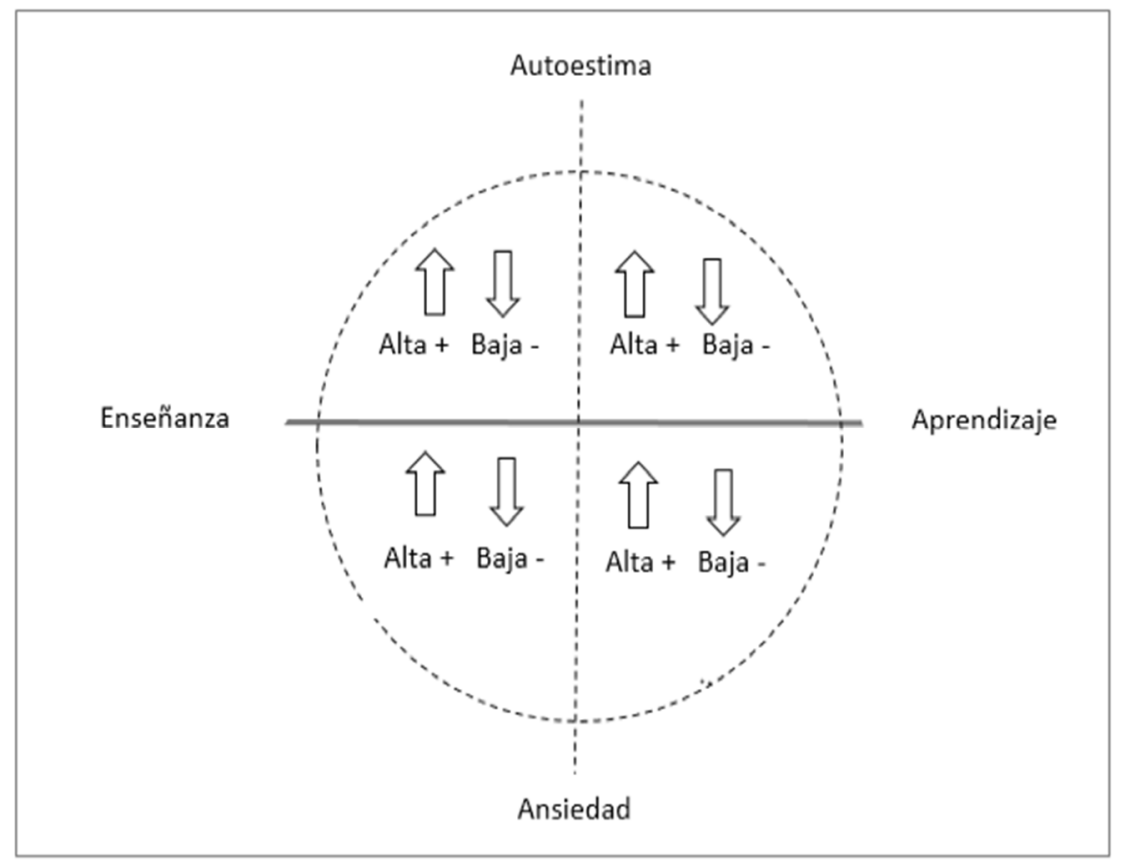

Figura 1: Modelo de relaciones entre autoestima y ansiedad

Fuente: Elaboración propia

El modelo representa las relaciones entre autoestima y ansiedad. Nuestra hipótesis es que estas relaciones pueden ser directas o inversas. En la figura 1 se ilustran las posibles relaciones que se presentan en la interacción social, específicamente en la relación pedagógica. Así, por ejemplo, un estudiante puede tener alta autoestima y presentar un alto grado de ansiedad, como sucede en aquellas personas que son muy exigentes consigo mismas. De la misma manera, se puede tener una alta autoestima y un nivel de ansiedad medio o bajo. Es importante aclarar que la autoestima guarda una relación con otros factores y no está simplemente determinada por aspectos relacionados a la ansiedad. Establecer las correlaciones que se dan entre los diferentes factores socio-afectivos en el proceso de aprendizaje puede ser de gran ayuda para que el docente implemente acciones en sus prácticas, metodologías y ambientes de trabajo que le permitan alcanzar el equilibrio entre estas y el aspecto cognitivo, y así movilizar actitudes y potencializar las habilidades de sus estudiantes en el aprendizaje de una lengua.

\section{Una aproximación a la ansiedad y sus tipologías}

Hasta aquí hemos dicho que establecer la importancia de los factores socio-afectivos en el aprendizaje de un idioma es una tarea muy compleja. Ahora bien, en el caso de la ansiedad, este componente ha sido catalogado como un elemento de incidencia negativa, es decir, puede llegar ocasionar dificultades en el aprendizaje. Estudios realizados a partir de la psicología resaltan el efecto perjudicial que tiene este factor. Al respecto, Pérez Paredes (2000) aborda el término ansiedad como un sentimiento negativo con causa desconocida que genera a quien lo padece desazón, y produce resultados negativos en la actividad que esté realizando. Asimismo, Rojas (2014) la define: "una emoción de alarma que da lugar a una hiperactivación fisiológica, donde todo se vive con miedos y temores y malos presagios" (Rojas, 2014, p. 26). Además de su significado, Rojas (2014) y Badilla \& Morales-Rojas (2016) afirman que este factor se induce por cuatro componentes: respuestas físicas, de conducta, cognitivas y sociales. El factor socio-afectivo afecta sin duda alguna nuestra forma de actuar y se exterioriza con algún estado emocional de miedo, bloqueo, irritabilidad, tensión entre otras. 
REVISTA DE LA ESCUela de CIENCIAS DE LA EDUCACIÓN, AÑo 15, NRO. 14, VOL. 1, ENERO A JUNIO DE 2019. PÁGINAS 26-35. ISSN 1851-6297 (DESDE DICIEMBRE DE 2006 A DICIEMBRE DE 2017). ISSN 2362-3349 (EN LINNEA). INCIDENCIA DE LOS FACTORES SOCIO-AFECTIVOS EN EL APRENDIZAJE DEL INGLÉS. LEYDY FERNANDA BONILLA JOJOA · MARIO DÍAZ VILLA.

Ahora bien, el concepto de ansiedad se asocia a una serie de sentimientos negativos, los cuales obstaculizan el proceso de aprendizaje. Horwitz y Cope (1986) fueron los primeros en plantear una teoría sobre a la ansiedad al momento de aprender una lengua extranjera. Ellos la definieron como:

Un fenómeno complejo, distinto de la ansiedad general, que se relaciona con el auto-concepto, las creencias, los sentimientos y los comportamientos que aparecen en una clase de idioma y que provienen de la unicidad del proceso de aprendizaje de idioma, puede provocar comportamientos específicos relacionados con la clase, un bloqueo (Horwitz y Cope, 1986, p. 126)

Sin embargo, algunos autores como Durlay, Burt \& Krashen (1982), consideran la ansiedad como un componente que puede llegar a ser favorable para el aprendizaje, y que genera una actitud positiva frente el mismo. Esto puede ocurrir cuando el docente planea actividades de baja exigencia que permiten que todos estudiantes logren realizarla de manera satisfactoria. En este caso se provoca en los estudiantes una motivación y una mejor autoestima frente al aprendizaje, pues ellos pueden llegar a sentir que si tiene capacidades y actitudes para hacerlo. Oxford (2000) plantea que está en manos del docente, y en las estrategias que implemente para el desarrollo de las actividades, lograr minimizar las dificultades que provoca la ansiedad en el aprendizaje.

Alpert \& Haber (1960) señalaron la distinción entre la ansiedad que genera un impacto positivo en los estudiantes y la catalogan dentro de una tipología de ansiedad facilitadora, y la que desencadena una sensación que perturba el proceso de aprendizaje. Esta ultima una ansiedad debilitadora. Krashen (1983), autor de la teoría "El Filtro Afectivo", apunta que las "variables afectivas" en el aprendizaje de las lenguas extranjeras puede jugar un papel facilitador o, por el contrario, dificultar dicho proceso de aprendizaje. De acuerdo con lo planteado por Krashen, el aula de clase debe contar con un "clima humano" en el cual se tengan en cuenta aspectos como la personalidad del alumno, la motivación del mismo, la autoestima, la confianza en sí mismo y la ansiedad, debido a que estos factores determinan si el aprendizaje se puede dar de manera óptima o, por el contrario, lo dificultan debido al bloqueo que pueden llegar a causar en los estudiantes.

Ahora bien, Maclntyre \& Gardner (1991) distinguen tres tipos de ansiedad a saber:

Ansiedad de rasgo: es un patrón característico que hace parte de la personalidad del individuo quien padece y constante en su diario vivir.

Ansiedad de estado: se presenta momentáneamente cuando el estudiante se ve inmerso en algún tipo de actividad o metodología.

Ansiedad específica/situacional: como su nombre lo indica, se presenta en situaciones de máxima exigencia para el estudiante (como una evaluación oral o escrita).

Las explicaciones precedentes han corroborado que la ansiedad tiene relación con el aprendizaje de las lenguas extrajeras. Horwitz \& Cope (1986) contemplaron que la ansiedad con respecto al uso de una lengua extranjera se evidenciaba en tres aspectos:

El miedo a interactuar en una lengua distinta a la materna.

El miedo a fracasar en las pruebas.

El miedo a una evaluación negativa.

En ese orden de ideas, dentro de su diseño de escala para medir la ansiedad en lenguas extranjeras Horwitz y Cope (1986) incluyeron estos tres aspectos como componentes a tener en cuenta para analizar dicho constructo. De alguna forma, la ansiedad influye en el rendimiento de los estudiantes, debido a que en muchas ocasiones el temor o la angustia impiden su participación. Esto repercute en los alcances o resultados dentro del proceso de aprendizaje de la lengua. A partir de este planteamiento Arnold (2000) señala que:

El profesorado tiene en sus manos dos opciones para aminorar la ansiedad del alumnado: eliminar la causa cuando sea posible, o proporcionar ayuda para enfrentarse a ella. Se puede reducir mucho la ansiedad por medio de la actitud del profesor y de la atmósfera que este crea en el aula (Arnold, 2000, p.16).

El profesor cumple un papel fundamental en el acto pedagógico, ya que puede generar prácticas y estrategias que permita a sus estudiantes tener un ambiente adecuado para el aprendizaje de la lengua. En este mismo sentido, Criado \& Mengual (2017) afirman que: 
REVISTA DE LA ESCUELA DE CIENCIAS DE LA EDUCACIÓN, AÑo 15, NRO. 14, VOL. 1, ENERO A JUNIO DE 2019. PÁGINAS 26-35. ISSN 1851-6297 (DESDE DICIEMBRE DE 2006 A DICIEMBRE DE 2017). ISSN 2362-3349 (EN LINNEA). INCIDENCIA DE LOS FACTORES SOCIO-AFECTIVOS EN EL APRENDIZAJE DEL INGLÉS. LEYDY FERNANDA BONILLA JOJOA · MARIO DÍAZ VILLA.

Además, al proporcionar la estimulación para crear un ambiente emocional y cooperativo positivo en el aula, los profesores [...] pueden contribuir a equipar a los estudiantes con herramientas necesarias para abordar el miedo a la evaluación negativa y la comunicación aprensión, especialmente en actividades basadas en el desempeño (Criado \& Mengual , 2017, p.31).

Independientemente del tipo de ansiedad que llegue a padecer el estudiante, el maestro debe facilitar el alcance de la lengua meta en sus estudiantes. Para ello debe reconocer que el acto comunicativo debe realizarse en un contexto que garantice la confianza y el deseo de aprender. De lo contrario puede inhibirse el proceso cognitivo del alumno y generar sentimientos que obstaculicen su aprendizaje. Chacón \& Hernández (2010) plantean que al no presentarse un vínculo socio-afectivo positivo con el docente y al no ser agradables las condiciones de aprendizaje para el estudiante, se puede afectar el rendimiento en el aprendizaje de la lengua.

Para concluir, es posible plantear que la ansiedad puede percibirse como un elemento negativo capaz de perturbar y dificultar no solo los procesos cognitivos, sino también los socio-afectivos (emocionales). Sin embargo, los estudios realizados en relación con la ansiedad han determinado que este factor no incide directamente de forma negativa en toda situación académica. Es el caso de la ansiedad facilitadora (en actividades que demandan bajo nivel de complejidad y promueve la participación) la cual no llega a perjudicar el rendimiento de los aprendices, sino que, por el contrario, estimula el alcance de la meta propuesta.

A lo largo del artículo se han presentado algunos factores socio-afectivos que inciden en el aprendizaje de la lengua extranjera. Estos juegan un papel trascendental, debido a que generan estímulos positivos o negativos en todas las actividades de vida, en general, y en el aspecto académico, en particular, y permiten que el aprendiz pueda alcanzar los objetivos propuestos y avanzar en su desarrollo intelectual, sin señalar un área específica de conocimiento a la cual éste deba enfrentarse.

En síntesis, la motivación, la autoestima y las demás actitudes que lleguen a adoptar los aprendices son fundamentales al momento de precisar si el aprendizaje ha sido exitoso o no (Dörnyei y Hadfield, 2014). Los factores socio-afectivos (motivación, autoestima y ansiedad) analizados guardan una relación, ya que la motivación tiene un vínculo con los factores personales, entre ellos, la autoestima. Ahora bien, al momento de presentarse inestabilidad en estos se pueden provocar sentimientos de angustia 0 desasosiego en la parte emocional. No obstante, la relación que media la autoestima y la ansiedad no es siempre tiene un carácter negativo.

Es necesario que el docente reconozca la importancia de los factores socio-afectivos y contextuales el proceso de los aprendices, e incorpore dentro de su labor estrategias flexibles que permitan desarrollar un ambiente vivencial para que el aprendizaje sea más significativo. Tanto los docentes como los demás actores dentro del sistema educativo deben ser conscientes que los estudiantes son seres integrales, es decir, que requieren desarrollar cuatro dimensiones: saber, saber hacer, saber ser y finalmente saber vivir juntos (Delors, 1994).Finalmente, los factores socio-afectivos están determinados por factores contextuales y juegan un papel significativo dentro de la vida de los aprendices y son determinantes al momento de trazar un objetivo en su vida personal y/o académica. Sin embargo, de la misma manera, éstos pueden generar una limitación o inhibición del desarrollo de las habilidades de los alumnos convirtiéndose en obstáculo para el alcance de las metas propuestas.

\section{Notas bibliográficas}

(1) Dörney (1994) plantea una clasificación de la motivación en tres niveles: en el nivel 1 de la lengua está asociada con la habilidad lingüística, el aprendizaje y objetivos de la misma, en otras palabras, comprende las razones que impulsan a los estudiantes a interesarse por la lengua. El nivel 2 del aprendiz está ligado a sus rasgos distintivos en términos de personalidad y motivación hacia la lengua. Finalmente, el nivel 3 de la situación de aprendizaje comprende factores internos y externos en relación con el contexto pedagógico tales como: el curso, el profesor y el grupo.

(2) Deci \& Ryan (2000) tipifican 4 tipos de regulaciones externas: la primera responde solo a estímulos externos y obedece a teorías operantes. La segunda regulación es la introyectiva y está relacionada con la presión generada para lograr que la persona motivada demuestre su habilidad. La tercera regulación identificada es la que ocurre cuando se considera que la conducta es el camino para llegar al objetivo, en otras palabras, hay un reflejo de conciencia sobre una conducta, aunque no le brinde sentimientos de placer o goce lo lleva al alcance de la meta propuesta. Por último, se encuentra la regulación integrada. Ésta sucede cuando hay una asimilación de las conductas identificadas y se internalizan en el "yo" dando buenos resultados. No obstante, puede ser considerada una motivación intrínseca, pero está catalogada dentro de lo extrínseco porque se centra en resultados y no en el disfrute o el interés por el aprendizaje.

(3) Mercer \& Williams (2014) establecen una diferencia entre autoestima y auto-concepto, ya que consideran que el auto-concepto es el resultado de la evaluación que realiza la autoestima sobre sí mismo en relación a los factores mencionados. 
REVISTA DE LA ESCUeLA dE CIENCIAS DE LA EDUCACIÓN, AÑo 15, NRO. 14, VOL. 1, ENERO A JUNIO DE 2019. PÁGINAS 26-35. ISSN 1851-6297 (DESDE DICIEMBRE DE 2006 A DICIEMBRE DE 2017). ISSN 2362-3349 (EN LINNEA). INCIDENCIA DE LOS FACTORES SOCIO-AFECTIVOS EN EL APRENDIZAJE DEL INGLÉS. LEYDY FERNANDA BONILLA JOJOA · MARIO DÍAZ VILLA.

\section{Referencias bibliográficas}

- Abascal, E. F., Cantero, F. P., \& Martínez-Sánchez, F. (2002). Introducción a la psicología de la motivación y la emoción. Psicología de la motivación y emoción. McGraw-Hill/Interamericana, pp. 3-34.Madrid.

- Alpert, R., \& Haber, R. N. (1960). "Anxiety in academic achievement situations". The Journal of Abnormal and Social Psychology, 61(2), 207-215. Recuperado de: http://dx.doi.org/10.1037/ h0045464

- Arnold, J. \& Brown, H.D. (2006). "El aula de ELE: un espacio afectivo y efectivo". Actas del Programa de Formación para el Profesorado de Español como Lengua Extranjera 2005-2006, 256-283. Instituto Cervantes, Munich. Recuperado https://cvc.cervantes.es/ensenanza/biblioteca_ele/publicaciones_centros/PDF/munich_20052006/03_arnold.pdf

- Arnold, J. (Ed.). (2000). La dimensión afectiva en el aprendizaje de idiomas. Cambridge University Press, Cambridge.

Recuperado:https://cvc.cervantes.es/ensenanza/biblioteca_ele/publicaciones_centros/PDF/rabat_2015/03_ arnold.pdf

- $\quad$ Badilla, D. C., \& Rojas, Y. M. (2016). "Estrategias para reducir la ansiedad durante las actividades orales en estudiantes de inglés". Revista de Lenguas Modernas, (25). DOI: https://doi.org/10.15517/rlm.v0i25.27705

- $\quad$ Bandura, A. (2001). "Social cognitive theory". An agentic perspective. Annual review of psychology, 52(1), pp. 1-26.

- Carrón, I. S. (2013). "Se me dan mal los idiomas" Aspectos psicológicos que pueden influir en la adquisición de lenguas extranjeras". Didáctica. Lengua y Literatura, 25, 275-293. Recuperado: http://revistas.ucm.es/index.php/DIDA/article/viewFile/42245/40219

- $\quad$ Chacón, G. P., \& Hernández, D. E. J. (2010). "El efecto del filtro afectivo en el aprendizaje de una segunda lengua". Letras, 2 (48), 209-225. Recuperado: https://dialnet.unirioja.es/servlet/ articulo?codigo=5476250

- Clément, R. (1980). "Ethnicity, contact and communicative competence in a second language". Language, pp. 147-154

- Covington, M. V. (1992). Making the grade: A self-worth perspective on motivation and school reform Cambridge University Press. Cambridge.

- $\quad$ Deci, E. L. \& Ryan, R. M. (2000). "Self-determination theory and the facilitation of intrinsic motivation, social development, and well-being". American psychologist, 55 (1), 68. DOI: 10.1037110003-066X.55.1.68

- Delors, Jacques .(1994) "Los cuatro pilares de la educación". La Educación encierra un tesoro. El Correo de la UNESCO, pp. 91-103. México.

- Dörnyei, Z. (1994). "Motivation and motivating in the foreign language classroom". The Modern language journal, 78(3), 273-284. http://dx.doi.org/10.2307/330107

- Dörnyei, Z. (1998). "Motivation in second and foreign language learning". Language Teaching, 31(3), 117135. DOI:10.1017/S026144480001315X

- Dörnyei, Z. (2008). Estrategias de motivación en el aula de lenguas.(Vol. 108) Editorial UOC, Barcelona.

- Dörnyei, Z. (2010). "Researching motivation: From integrativeness to the ideal L2 self". Introducing Applied Linguistics: Concepts and Skills, 3(5), 74-83.

- Dörnyei, Z., \& Hadfield, J. (2014). Motivating learning. Routledge and Kegan Paul, London.

- Dörnyei, Z., \& Ushioda, E. (2013). Teaching and Researching: Motivation. Routledge and Kegan Paul, London.

- $\quad$ Dulay, H., Burt, M., \& Krashen, S. (1982). Language two. Oxford University Press, London.

- Gardner, R. C. (2010). Motivation and Second Language Acquisition: The socio-educational mode/Vol. ) Peter Lang, New York . (10

- $\quad$ Gardner, R.C. (1985). Social Psychology and Second Language Learning. Arnold Publishers, London.

- Gardner, R.C. y Lambert, W.E. (1972), Attitudes and Motivation: Second Language Learning Massachusetts: Newbury House Publishers.

- Hilgard, E. R., Atkinson, R. C., \& Atkinson, R. L. (1979). Introduction to Psychology. Harcout Brace Jovanovich. Inc. I., New York.

- Horwitz, E. K. (1990). "Attending to the affective domain in the foreign language classroom". Shifting the instructional focus to the learner, pp. 15-33.

- Horwitz, E. K., Horwitz, M. B., \& Cope, J. (1986). "Foreign language classroom anxiety". The Modern Language Journal, 70(2), pp. 125-132. Recuperado: https://doi.org/10.1111/j.1540-4781.1986.tb05256.x

- Koosha, B., Ketabi, S., \& Kassaian, Z. (2011). "The Effects of Self-esteem, Age and Gender on the Speaking Skills of Intermediate University EFL Learners". Theory \& Practice in Language Studies, 1(10). Recuperado: https://www.researchgate.net/publication/267372651 The Effects of Selfesteem_Age_and_Gender_on_the_Speaking_Skills_of_Intermediate_University_EFL_Learners.

- Krashen, S. (1985). The Natural Approach: Language Acquisition in the Classroom. Prentice Hall, New York.

- Maclntyre, P. D., \& Gardner, R. C. (1991). "Language anxiety: Its relationship to other anxieties and to processing in native and second languages". Language learning, 41(4), pp. 513-534.

- Marins De Andrade, P. R., \& Guijarro Ojeda, J. R. (2010). "Afectividad y competencia existencial en estudiantes de español como lengua extranjera en Brasil". RLA. Revista de lingüística teórica y aplicada, 48(1), pp. 51-74. Recuperado: http://dx.doi.org/10.4067/S0718-48832010000100004

- Maslow, A. (1990). La personalidad creadora. Kairós, Barcelona.

- Maslow, A. (2016). El hombre autorrealizado: hacia una psicología del ser. Editorial Kairós, Barcelona.

- $\quad$ Mercer, S., \& Williams, M. (Eds.). (2014). Multiple perspectives on the self in SLA. Multilingual Matters, 
REVISTA DE LA ESCUELA DE CIENCIAS DE LA EDUCACIÓN, AÑo 15, NRO. 14, VOL. 1, ENERO A JUNIO DE 2019. PÁGINAS 26-35. ISSN 1851-6297 (DESDE DICIEMBRE DE 2006 A DICIEMBRE DE 2017). ISSN 2362-3349 (EN LINNEA). INCIDENCIA DE LOS FACTORES SOCIO-AFECTIVOS EN EL APRENDIZAJE DEL INGLÉS. LEYDY FERNANDA BONILLA JOJOA · MARIO DÍAZ VILLA.

Toronto.

- Murado Bouso, J. L. (2010). Didáctica de inglés en educación infantil.Métodos para la enseñanza y el .aprendizaje de la lengua inglesa. Ideas propias, Vigo

- $\quad$ Navarro-Pablo, M., \& Jiménez, E. G. (2018). "Are CLIL Students More Motivated? An Analysis of Affective Factors and their Relation to Language Attainment". Porta Linguarum: Revista Internacional de Didáctica de las Lenguas Extranjeras, (29), pp. 71-90.Recuperado de: https://dialnet.unirioja.es/servlet/articulo?codigo $=6273211$

- $\quad$ Noels, K. A., Pelletier L. G, Clément, R. \& Vallerand R. J. (2002). 'Why Are You Learning a Second Language? Motivational Orientations and Self-determination Theory'. Language Learning. 50 (1): pp. 5785. Recuperado de: https://onlinelibrary.wiley.com/doi/abs/10.1111/0023-8333.00111

- Oxford, R. (2000): "La ansiedad y el alumno de idiomas: nuevas ideas", en J. Arnold (Ed.) La dimensión afectiva en el aprendizaje de idiomas, 77-86. Cambridge University Press, Madrid.

- Peiteado, M. G., \& López, B. R. (2017). "Factores motivacionales de los adultos para el estudio de una lengua extranjera". Pedagogía social: Revista Interuniversitaria, (30), 129-141. DOI:10.7179/PSRI 2017.30.09.

- Pérez Paredes, P. F. (2000). Actividades comunicativas y su relación con la generación de ansiedad en estudiantes de inglés como lengua extranjera. Universidad de Murcia, Servicio de publicaciones, Murcia.

- Rodríguez-Pérez, N. (2012). "Causas que intervienen en la motivación del alumno en la enseñanzaaprendizaje de idiomas: el pensamiento del profesor". Didáctica. Lengua y literatura, 24, 381409.Recuperado: http://revistas.ucm.es/index.php/DIDA/article/viewFile/39932/38396

- $\quad$ Rojas, E. (2014). Cómo superar la ansiedad: la obra definitiva para vencer el estrés, las fobias y las obsesiones. Editorial Planeta, S. A, Barcelona.

- Rubio-Alcalá, F. (2017). "The links between self-esteem and language anxiety and implications for the classroom". In Gkonou, Christina, Mark Daubney and Jean-Marc Dewaele (eds.) New Insights into Language Anxiety: Theory, Research and Educational Implications. Multilingual Matters, pp. 198-216, Bristol.

- $\quad$ Serra, D. J. G. (2008). Psicología de la motivación. Editorial Ciencias Médicas, La Habana.

- Vallerand, R. J. (1997). "Toward a Hierarchical Model of Intrinsic and Extrinsic Motivation". En M. P. Zanna (ed.), Advances in Experimental Social Psychology, 271-360. Academic Press, New York.

- Vázquez, I. N., \& Pérez, R. C. (2016). "La ansiedad como variable predictora de la autoestima en adolescentes y su influencia en el proceso educativo y en la comunicación". Revista Ibero-americana de Educación, 71(2), pp. 109-128

- Villegas, J. D. C. S., García-Santillán, A., \& Escalera-Chávez, M. E. (2016). "Variables que influyen sobre el aprendizaje del inglés como segunda lengua". Revista Internacional de Lenguas Extranjeras, (5), 79-94. Recuperado: https://www.raco.cat/index.php/RILE/article/view/316766

- Weiner, B. (1979). "A theory of motivation for some classroom experiences". Journal of Educational Psychology, 71(1), 3.

- Zare, P., \& Riasati, M. J. (2012). "The relationship between language learning anxiety, self-esteem, and academic level among Iranian EFL learners". Pertanika Journal of Social Sciences and Humanities, 20(1), pp. 219-225 